\title{
Physiological roles of dietary glutamate signaling via gut-brain axis due to efficient digestion and absorption
}

\author{
Kunio Torii $\cdot$ Hisayuki Uneyama $\cdot$ Eiji Nakamura
}

Received: 4 February 2013/Accepted: 4 February 2013/Published online: 6 March 2013

(C) Springer Japan 2013

\begin{abstract}
Dietary glutamate (Glu) stimulates to evoke the umami taste, one of the five basic tastes, enhancing food palatability. But it is also the main gut energy source for the absorption and metabolism for each nutrient, thus, only a trace amount of Glu reaches the general circulation. Recently, we demonstrated a unique gut sensing system for free Glu (glutamate signaling). Glu is the only nutrient among amino acids, sugars and electrolytes that activates rat gastric vagal afferents from the luminal side specifically via metabotropic Glu receptors type 1 on mucosal cells releasing mucin and nitrite mono-oxide (NO), then NO stimulates serotonin (5HT) release at the enterochromaffin cell. Finally released 5HT stimulates $5 \mathrm{HT}_{3}$ receptor at the nerve end of the vagal afferent fiber. Functional magnetic resonance imaging (f-MRI, 4.7 T) analysis revealed that luminal sensing with $1 \%(\mathrm{w} / \mathrm{v})$ monosodium L-glutamate (MSG) in rat stomach activates both the medial preoptic area (body temperature controller) and the dorsomedial hypothalamus (basic metabolic regulator), resulting in diet-induced thermogenesis during mealing without changes of appetite for food. Interestingly, rats were forced to eat a high fat and high sugar diet with free access to $1 \%$ (w/w) MSG and water in a choice paradigm and showed the strong preference for the MSG solution and subsequently, they displayed lower fat deposition, weight gain and blood leptin. On the
\end{abstract}

K. Torii · H. Uneyama · E. Nakamura

Institute for Innovation, Ajinomoto Co., Inc.,

1-1 Suzuki-cho, Kawasaki-ku, Kawasaki,

Kanagawa 210-8681, Japan

Present Address:

K. Torii $(\bowtie)$

Torii Nutrient-Stasis Institute, Inc., Miyuki Building,

5-6-12 Ginza, Chuo-ku, Tokyo 104-0061, Japan

e-mail: kuniotorii@toriilab.com other hand, these brain functional changes by the f-MRI signal after $60 \mathrm{mM}$ MSG intubation into the stomach was abolished in the case of total vagotomized rats, suggesting that luminal glutamate signaling contributes to control digestion and thermogenesis without obesity.

Keywords Glutamate signaling · Gastric vagal afferent · Functional MRI - Diet induced thermogenesis · Gut-brain axis · Digestive juice secretion

\section{Introduction}

Chemical senses during a meal are a cue to recognize food ingestion and to determine its composition of nutrients when chemical substances stimulate sensory organs. Olfaction and taste are typically known as chemical senses, and subsequent visceral senses by digested products in the alimentary tract is as an intake signal of nutrients which were absorbed into the inner body sensory organs such as blood vessels, lymph node, liver, kidney, and brain. Major physiological roles of chemical senses include recognition of food intake, smooth digestion and absorption, and maintenance for homeostasis of each nutrient in the blood as well as the brain after a meal. Additionally, regulation of glucose and free fatty acid concentration in the blood and related hormones released from specific tissues can also primarily be occurring by chemical senses for control of appetite and satiety for foods intake as a major marker for energy balance in the living body.

The concentration of each amino acid in the blood as well as the brain are metabolically controlled within certain limits all day long, regardless of the amount and the quality of meals containing any dietary protein. As such, attention is focused on visual sense, texture, olfaction and taste 
during the meals, to judge whether it is food or not, which nutrients are included in what amount, and whether there has been some changes from past experience, retrieving previous memory. Once the brain judges there would be no problem based on the past experience of similar food, the esophagus opens and food is swallowed with saliva, which includes electrolytes, and other nutrients such as amino acids and digestive enzyme such as $\alpha$-amylase that digests starch to amylose. A aense of taste profile adapts to intake these nutrients and forms boundary concentrations to feel the particular taste such as sweetness for energy, saltiness for electrolytes, and umami taste for protein. In other words, in order to feel the taste of food, in parallel with the composition of nutrients, they must be more concentrated rather than those included in the saliva, which is the reason that foods rich in nutrients but have no smell or taste are hard either to stimulate appetite or to reach satiety. For example, we can eat cooked rice which has little taste, because we used to get it. But actually we were able to eat the rice with other palatable food although it is not easy to eat the rice alone.

Recent research has exposed that L-glutamate (Glu), an amino acid, has multiple physiological functions in our body during and after a meal. As an essential substrate in the intermediary metabolism, therefore, free Glu is present in most organs and tissues (skeletal muscles, brain, kidneys, and liver) in substantial concentrations [1, 2]. Glu plays an important role for energy metabolism and the substance for metabolism of other amino acids, glutathione, and body proteins. In the brain, Glu, which is locally produced completely de novo from glucose, acts as a major excitatory neurotransmitter, and its activity regulates synaptic plasticity, learning, memory, motor activity, and neural development. But dietary Glu is almost impermeable into the circulating blood. Also there is the bloodbrain barrier against Glu in between blood and brain so as to not to incorporate into it the brain. This Glu plays independently of dietary Glu in the alimentary tracts as a Glu signaling mainly, a major portion of Glu is metabolized for energy sources and partially for substrate for protein synthesis.

In the oral cavity, Glu in foods elicits a unique taste termed umami taste, which is generally thought to be a signal for dietary protein ingestion in the brain. In addition to the gustatory roles of Glu as a typical umami taste material, recent studies have unveiled the postingestive significance of Glu on various physiological functions such as the process of digestion, nutrient absorption and metabolism, and energy homeostasis via brain activation. These effects might be mediated via luminal gut Glu sensors functionally linked to the afferent branches of the vagus nerve as visceral sensations, or particularly via the afferent sensory nerves in the oral cavity as a taste sensation. Moreover, Glu acts as a reinforcer after ingestion via vagal afferent activation in the gut. For example, we recently observed that an intragastric infusion of $60 \mathrm{mM}$ Glu aqueous solution induced a conditioned flavor preference $(\mathrm{CFP})$ in rats $[3,4]$. It is strongly suggested that the sense of Glu mortified food intake into the brain to prepare to operate efficient digestion in parallel with memory functions due to expectation for normal appetite for foods and maintenance for homeostasis.

In recent years, umami taste was categorized as a basic taste like others, i.e., sweetness, saltiness, bitterness and sourness. Typical umami taste material is monosodium L-glutamate (MSG) that is generally used as seasoning of umami taste material beyond 2.5 million tons per year, and the consumption is still increasing by several percent per year. This fact strongly suggests that MSG plays physiological roles in the living body including humans during and after meals particularly in the alimentary organs as visceral information via the gut-brain axis except the umami sensation orally. Thus, this review was conducted to summarize recent progress in efficacies of Glu signaling in gut including gut-brain communication for a healthier life.

\section{Gustatory glutamate stimuli regulate autonomic nerve activity}

Taste sensations of MSG elicit various visceral efferent nerve activities and gut functions. Salivary secretion is one of the taste-induced autonomic reflexes [5]. The relative strengths of different stimuli inducing the parotid salivary flow are as follows: citric acid (sour) $\geq$ MSG (umami) $>\mathrm{NaCl}$ (salty) $>$ sucrose (sweet) $\geq$ magnesium sulfate (bitter) [6]. The role of saliva is not only to lubricate food for mastication and swallowing but also to initiate the digestion of nutrients, i.e., carbohydrates and fats, because it contains the digestive enzymes such as $\alpha$-amylase and lipase. In addition, there are various reports describing other taste-induced reflexes. Sweet taste stimulation with sucrose and glucose in iso-tonic solution increases the efferent activity of the pancreatic and the hepatic vagus nerves in rats, whereas a very salty taste solution containing a high concentration of $\mathrm{NaCl}$ suppresses such activity because of aversive foods stimuli for the expected disorder of homeostasis [7-10]. In addition, sweet taste stimulation elicits insulin release prior to increasing plasma glucose levels, a process called cephalic-phase insulin release [11-13]. By contrast, sweet taste stimulation was observed to suppress vagal gastric efferent (VGE) activity and the efferent activity of the adrenal, pancreatic and hepatic excitation of sympathetic nerves, whereas salty taste stimulation was shown to increase these activities [9, 
Fig. 1 Reflex activation of vagal gastric and pancreatic nerve activity stimulated through oral, gastric, intestinal and hepatoportal glutamate sensors. Reproduced from Niijima [17]

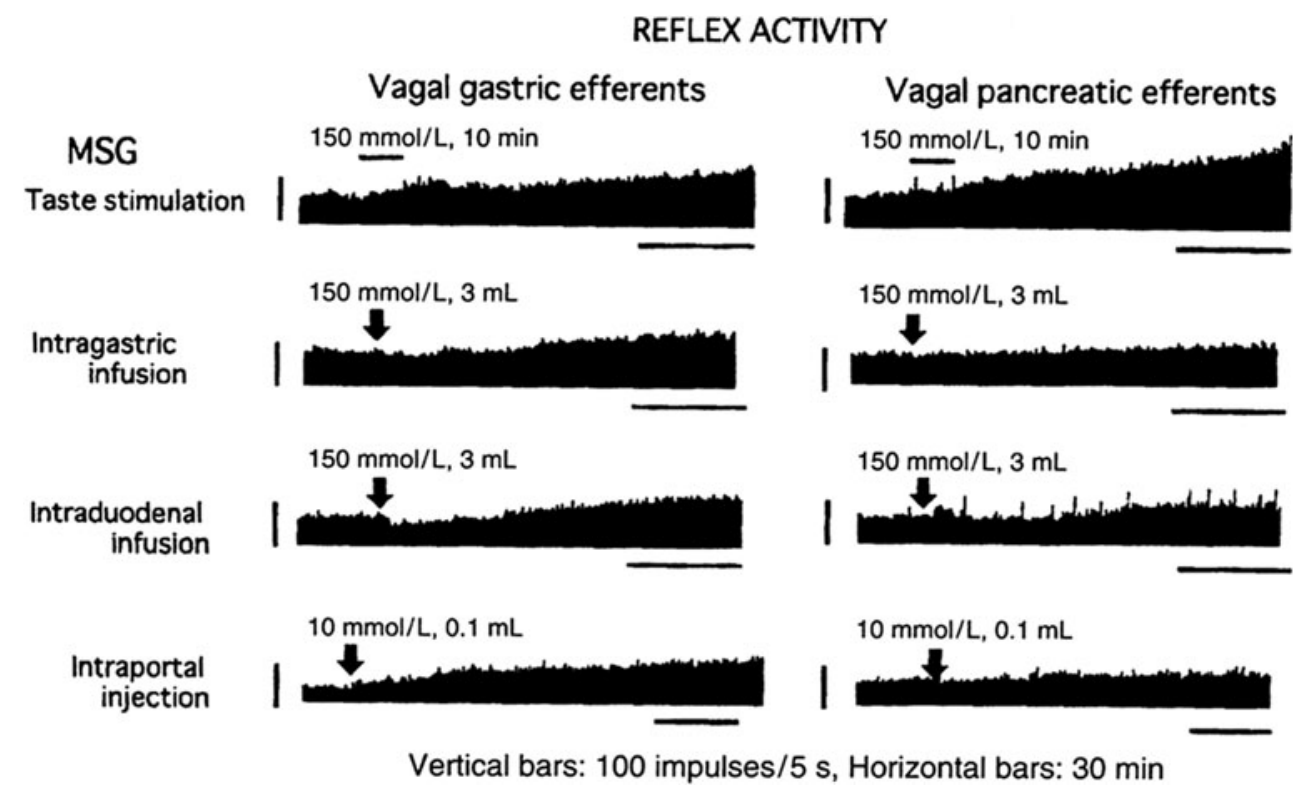

14]. Moreover, sweet taste signals stimulate gastric acid secretion ( $\mathrm{pH}$ 0.56) via the vagus nerve excitation [15].

Umami taste stimulation produced by a MSG isotonic solution $(150 \mathrm{mM})$, was able to activate VGE activity in overnight fasted rats and the efferent activity of the pancreatic and hepatic vagus nerves (Fig. 1) [10, 16, 17], in association with an increase in insulin secretion [18]. However, it has been reported that MSG aqueous solution without food ingredients does not elicit cephalic-phase insulin release [19], so further study to evaluate certain permissive effect will be necessary in the case of food intake with or without MSG. In addition, Glu stimulation orally enhanced the sympathetic efferent activity into the adipose tissue in overnight fasted rats, markedly suggesting that recognition of food intake by Glu signaling via vagal afferent excitation reduced lipolysis during food deprivation or overnight fasting.

\section{Gut nutrient stimuli control vagus nervous activity in the stomach during a meal}

In the gastrointestinal (GI) tract, various nutrients are detected and absorbed through the luminal layer. Nutrients also regulate the activity of vagal afferent nerves and the release of GI peptides, including cholecystokinin (CCK), peptide YY, glucagon-like peptide-1 (GLP-1) and -2, leptin, ghrelin, and others [20-23] due to efficient usage of adsorbed nutrients and their homeostasis in the living body after a meal.

It was thought for a long time that the vagal gastric afferents (VGA) in the stomach could detect only gastric distension and not individual nutrients as chemical senses. However, we have previously reported that Glu evoked

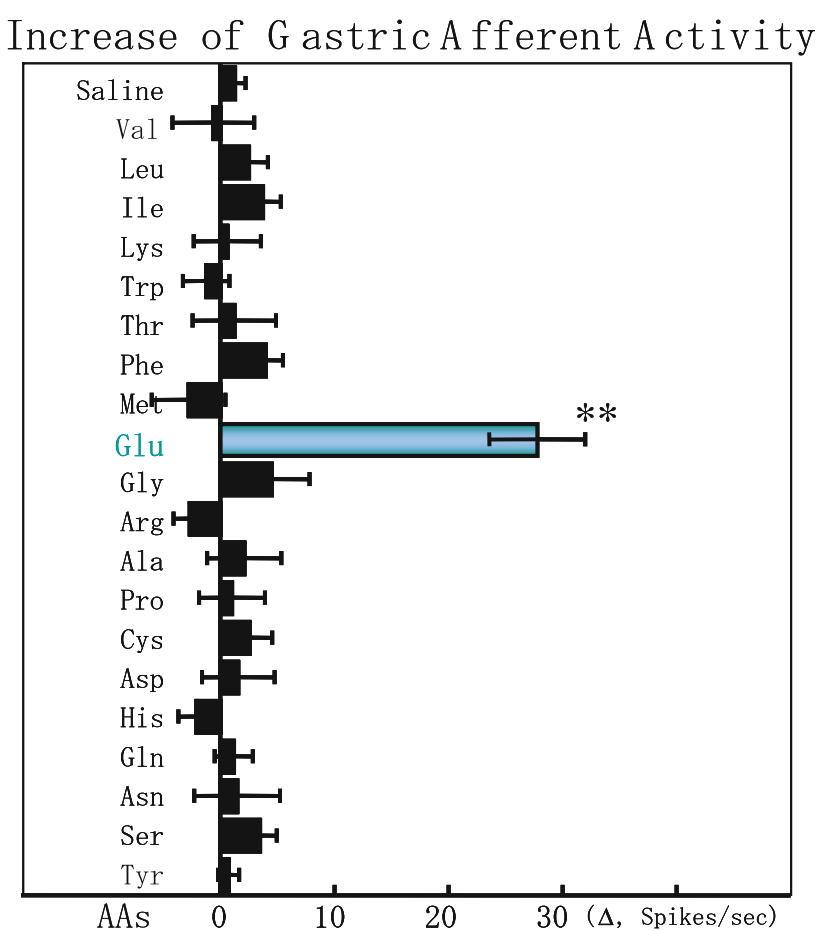

Fig. 2 Vagal gastric afferent (VGA) responses to intragastric infusion of various amino acid solutions. Each aqueous solution ( $150 \mathrm{mmol} / \mathrm{l}, 2 \mathrm{ml} / \mathrm{rat}$ ) was intubated to rat stomach, and the mean value of discharge rate above baseline at $20 \mathrm{~min}$ was plotted. Each column and horizontal bar represents mean \pm SEM from 5 rats. $* * p<0.05$ versus saline (Kruskal-Wallis test). Reproduced from Uneyama et al. [24]

visceral sensations in the stomach [17]. This is important in the field of gastric nutrient perception because these data strongly suggest that chemical perception, in particular an amino acid-sensing system, exists in the gastric mucosa. Interestingly, among the 20 kinds of amino acids, Glu 
alone stimulates the nerve end of rat VGA (Fig. 2) [24]. There are no responses to any sugars including glucose and sodium chloride, luminal perfusion in rats under the peripheral anesthetic treatment with lidocaine, abolished the Glu-evoked VGA activation, indicating that this response is a chemical event within the tissue of gastric mucosa. Furthermore, the Glu response was blocked by either depletion of serotonin (5-HT) using parachlorophenylalanine (PCPA) treatment for 5HT type $3\left(5-\mathrm{HT}_{3}\right)$ receptors in the nerve end of vagal afferent, or inhibition of the nitric oxide (NO) synthetase using L-NAME treatment. The vagal afferent response was also mimicked by luminal perfusion with an NO donor such as sodium nitroprusside. In addition, NO donor-induced afferent activation was abolished by $5-\mathrm{HT}_{3}$ receptor blockage [24]. This finding strongly supports the possibility of intercellular communication in the rat gastric mucosa between mucosal cells and the vagus nerve-end using $\mathrm{NO}$ and 5-HT as potent stimuli during digestion processes. More than $90 \%$ of 5-HT throughout the body is concentrated and localized in the enterochromaffin (EC) cells of the GI mucosa. The physiological role of mucosal 5-HT from EC cells serves a paracrine function by specifically recognizing Glu in the lumen of the stomach, which is similar to the physiological role, as reported in the duodenal glucose sensing system.

\section{Chemical senses in alimentary tracts to regulate metabolism of absorbed nutrients}

Our understanding of the sensing of nutrients in the gut, luminal layer may contains particular cells to be referred to as an "intestinal sensor cell" as originally proposed in the 1970s by Fujita et al. [25] This hypothesis suggests that nutrient-sensing cells are distributed in the gastric antrum or duodenal mucosa and that when these cells interact with luminal nutrients in the digested foods, they enhance to release hormones in an endocrine or paracrine manner to transfer information about luminal nutrient content to other organs, including the brain either via endocrine or vagal pathways. However, these cells involved in the gut nutrient perception system remained to be identified for a long time. In 1996, Höfer et al. [26] reported that taste-like cells similar to the taste cells in the oral cavity are distributed in the gastric and intestinal mucosa and they proposed that these taste-like cells represent the unknown sensor cells. Subsequently, with the development of molecular biology techniques in the field of taste research, several taste receptors responding to different amino acids have been identified. We now know that metabotropic Glu receptors (mGluRs), a calcium sensing receptor (CaSR), and a taste receptor (a heterodimer of T1R1 and T1R3: T1R1/T1R3) are all linked to amino acid sensation in the tongue. These same receptors are also candidates for luminal amino acid sensors. Although the molecule(s) that sense Glu in the gastric mucosa is/are still unclear, intragastric infusion of MSG causes a vago-vagal reflex, which increases VGE, as well as vagal pancreatic and celiac efferent activities (Fig. 1) [17, 27]. Interestingly, inosine $5^{\prime}$-monophosphate, another umami substance that enhances MSG binding to taste receptors and thus enhances umami taste intensity synergistically, also activates VGA and increases vagal celiac efferent activity [27] in an additional manner. Assuming the coexistence of free Glu with dietary protein is very common in foods, these findings suggest that a specific Glu-sensing system in the stomach could contribute to the gastric phase of protein digestion and could integrate nutrient information in the brain by vagal afferent excitation. We defined recently that the possible Glusensing system in the stomach is a metabotropic glutamate receptor type 1 (mGluR1) in mucosal cells that is to express NO synthetase to stimulate 5-HT release through the vagal afferents excitation. There is little contribution by EC cells expressing TIR1/TIR3 to induce 5-HT release $<5 \%$ in total [24]. Recently, we defined that free Glu concentration in saliva and pancreatic juice is several times higher ti than in its blood level. This digestive juice derived Glu possibly collaborates with dietary Glu to act as Glu signaling in alimentary tracts.

In contrast to what occurs in the small intestine, there are many reports that intraduodenal infusion of amino acids or oligo-peptides alters the vagal celiac afferent (VCA) activity. Sharma and Nasset [28] observed an apparent increase in the mesenteric afferent activity in either wholenerve or multifiber preparations from the GI tract following amino acid infusions in cats. Using a unitary recording technique in the nodose ganglion, Jeanningros and colleagues subsequently revealed in detail the response of VCA to various kinds of amino acid infusions in the cat small intestine. Their report described many sensors responsive to arginine, leucine, and other amino acids [29, 30]. Recently, we re-examined the luminal amino acid sensitivity of VCA in rats. Intraintestinal infusion of MSG, lysine, leucine, and other amino acids evoked excitatory responses in VCA [31]. In contrast to the responses in these amino acids, the same treatment with glycine, methionine, and certain other amino acids led to the depression of afferent nerve activity [31]. In rats, duodenal infusions of protein hydrolysates also increased mesenteric afferent activity [32, 33]. Schwartz and Moran [33] revealed that duodenal intubation of protein hydrolysates (e.g., peptone) stimulated vagal celiac afferents, indicating that an amino acid sensor or oligopeptide sensor might exist in the rat luminal duodenum. However, the mechanisms underlying such sensations are not fully understood, and further research is needed. 
Changes in the VCA activity induce autonomic reflexes and regulate various visceral functions, oral and intragastric and intestinal infusions of MSG isotonic solution in rats resulted in an increase in VGE, vagal pancreatic efferent activity [16, 17], and lysine evoked long-lasting enhancement of VGE activity in lysine-deficient rats [31]. On the other hand, the intraintestinal infusion of glycine inhibited VGE activity [31]. In addition, introduction of a glucose solution into the intestine increased VCA activity; the sensing mechanism underlying glucose effects has been described in another review [34]. A glucose solution also suppressed sympathetic adrenal efferent activity and enhanced vagal pancreatic efferent activity [31]. These observations support our hypothesis that the vagal GI afferent signals, particularly gastric branch by Glu signaling, are to recognize food intake, regulate gastric emptying, GI motility, metabolic control for homeostasis and both appetite and satiety for food $[35,36]$.

\section{Brain activation after gut nutrient stimulation}

From our recent studies, in addition to autonomic reflexes, the effects of ingested nutrients in the alimentary tracts are processed in the forebrain, which determines whether food is good or not for content of required nutrients and maintenance of homeostasis and subsequently regulates feeding behavior in a palatable manner. To investigate which regions of the rat brain respond to ingested nutrients, we used an functional MRI (fMRI, $4.7 \mathrm{~T}$ ) technique. The merit of fMR is that activated areas in the well-trained rat whole brain can be investigated simultaneously by non-invasive awake conditions without unpleasant stresses, like any painful treatment. An intragastric intubation of $60 \mathrm{mM}$ MSG, or isocaloric $(60 \mathrm{mM})$ glucose and an equimolar $\mathrm{NaCl}$ solution have been shown to activate distinct forebrain regions (Fig. 3) [37, 38]. An intragastric load of MSG significantly activated several brain regions including the hippocampus (memory function), the amygdala central body (preference center for nutrients), the lateral hypothalamus (feeding center), the dorsomedial hypothalamus (basic metabolism regulator), and the medial preoptic (body temperature regulator). On the other hand, an intragastric infusion of glucose activated the insular cortex, amygdala, nucleus accumbens (which is the terminal dopaminergic projection for indulgence or craving), and the lateral and ventromedial (satiety center) hypothalamus. We also investigated brain responses to an intragastric load of corn oil emulsion, which activated the amygdala, the lateral hypothalamus, the hippocampus, and the ventral tegmental area [39]. The changes of brain function after $60 \mathrm{mM}$ MSG intubation into the stomach to cause thermogenesis for energy expenditure as diet induced thermogenesis during and after a meal. There was a concern about prevention of obesity development, in the model of rats, fed a high sugar and fat diet for 1 year from the wearing period. Control animals offered to drinking water alone, became typically obesity but similar ones were
Fig. 3 Activated area of rat forebrain specific to the stimulation of treatment with post-oral nutrients (glucose, MSG, $\mathrm{NaCl} 60 \mathrm{mM}$ and $\mathrm{NaCl}$ $150 \mathrm{mM}$ ) compared with common control images which are averaged during $10 \mathrm{~min}$ before administration. T-map images (bregma +2.0) depict activated brain regions at preadministration ( $-5 \mathrm{~min}), 10$, 20 , and 40 min after the onset of intragastric infusion. Upper figure is a template image overlaid with Paxinos Atlas. $A C C$ anterior cingulate cortex, $C P u$ caudate putamen, $I C x$ insular cortex, $N A C$ nucleus accumbens. Color bar, $t$ value. Reproduced from Tsurugizawa et al. [37]

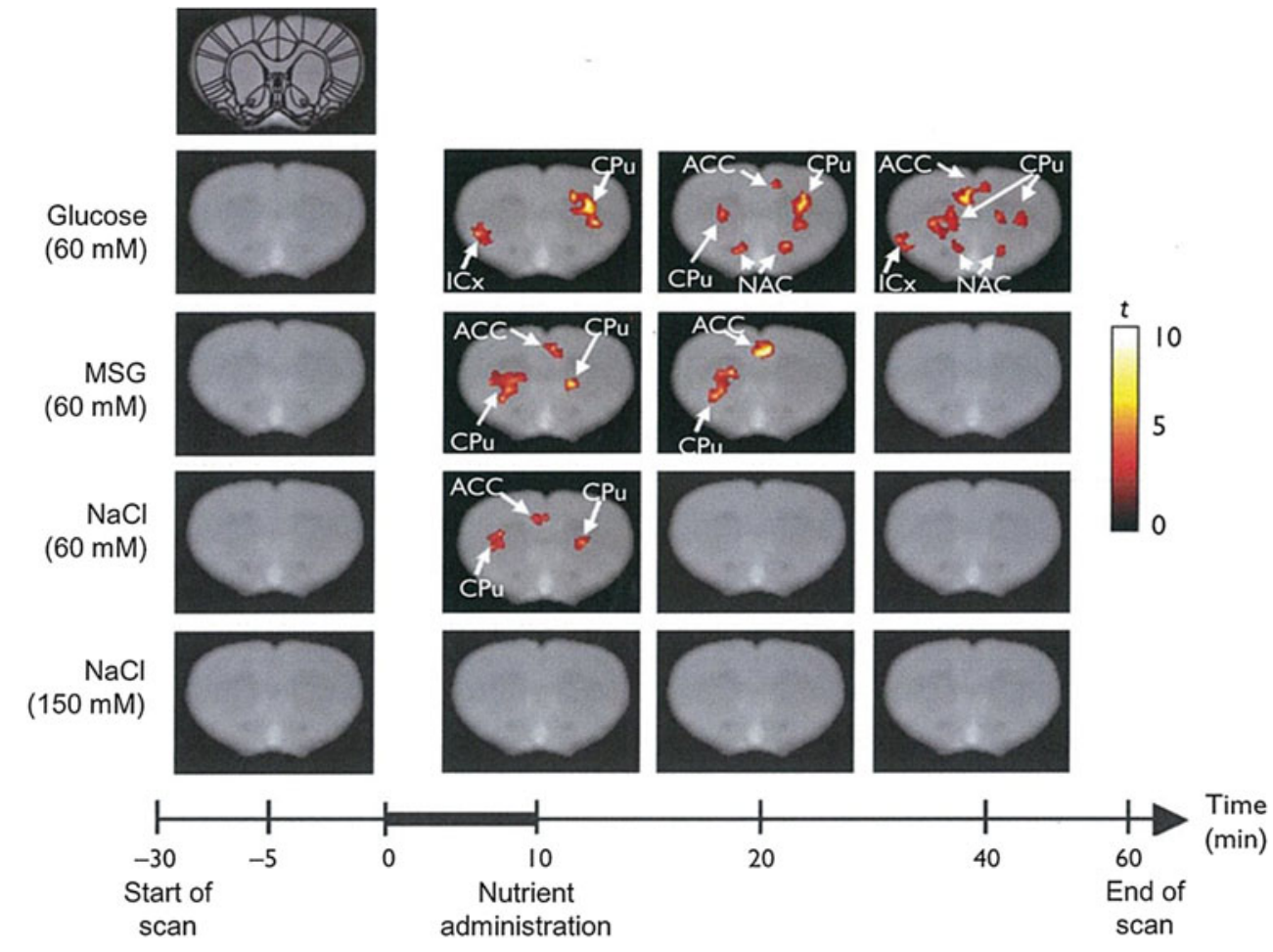


freely available to drink $1 \%(\mathrm{w} / \mathrm{v})$ MSG solution and water in a choice paradigm, and dominantly preferred the MSG solution [40]. Glu signaling during digestion processes may enhance thermogenesis and force excessive intake of every source from an experimental diet to be cured as energy expenditure suggesting that Glu is effective in not developing obesity when overeating occurs [38].

Behavioral studies also showed that ingested glutamate or glucose (60 $\mathrm{mM}$ in each) in water or corn oil emulsion have positive postingestive effects with regard to flavor preference learning in rats [3, 39, 41]. In rodents and humans, the preference for the flavor of an ingested solution can be increased by repeatedly pairing it with ingestion or intragastric infusion of certain solutions containing particular nutrients to be required physiologically. This paradigm is called CFP. Behavioral studies revealed that the intragastric infusion of liquid containing carbohydrates, lipids, and alcohols induces CFP in rodents [41, 42]. In addition, we previously showed that an intragastric load of $60 \mathrm{mM}$ MSG evoked CFP in rats. Isocaloric $(60 \mathrm{mM})$ glucose and equimolar $(60 \mathrm{mM}) \mathrm{NaCl}$ solution did not evoke CFP (Fig. 4) [3, 4], although $480 \mathrm{mM}$ hypertonic glucose aqueous solution did evoke CFP because of an unpleasant symptom occurring. Much higher concentrations of glucose lead to an increase in blood glucose and insulin release. These results indicate that the preference for the flavored solution paired with a gut infusion of MSG is coupled with memory function operation to establish CFP due to neither a caloric effect nor hyperosmotic pressure effect. Based on the results of functional brain imaging and CFP behavioral studies, the brain regions commonly activated in response to the intragastric infusion of either MSG or glucose in water $(60 \mathrm{mM})$ or corn oil emulsion include the anterior cingulate cortex, insular cortex, amygdala, caudate-putamen, hippocampus, and the lateral hypothalamus $[38,39]$. Thus, these regions should be related to CFP. In particular, the lateral hypothalamus is an important area for regulating intake of food or liquid containing nutrients.

A previous report revealed that lesions of the lateral hypothalamus diminished CFP induced by intragastric infusion of a glucose solution [43]. The glucose-sensitive neurons that exist in the ventromedial hypothalamus are activated as the intracellular glucose levels increase as a satiety signal. Dopaminergic projections from the ventrotegmental area to the nucleus accumbens, the amygdala, and the lateral hypothalamus are related to the preference for, or addiction to, ingested energy sources, such as glucose-like sugars and corn oil. Some studies showed that sugar intake increased dopamine release in the nucleus accumbens shell region in rats, reportedly causing them to become an addicted behavior to all sugars [44]. On the other hand, intragastric infusion of MSG and $\mathrm{NaCl}$ does not activate the nucleus accumbens (Fig. 3), and lesions of neurons in the ventrotegmental area bilaterally using 6 hydroxy dopamine do not affect the preference for MSG and $\mathrm{NaCl}$ in rats [45]. These results show that the postingestive effects of $\mathrm{Glu}$ and $\mathrm{NaCl}$ differ from those of sugars and lipids. Similar rats under lysine deficiency strongly preferred lysine solution with bitterness quantitatively in a choice paradigm among whole amino acids. Nevertheless, there were no differences with or without the ventrotegmental area lesioned. It is manifestthat any amino acid and $\mathrm{NaCl}$ are independent to indulgence and/or addiction like sugars and lipids.

Another advantage of fMRI is that it has better temporal resolution than an alternative monitoring technique, c-fos labeling. The time course of particular brain area activation is different for MSG and for glucose, as revealed by fMRI (Fig. 5) [37]. An intragastric infusion of $60 \mathrm{mM}$ MSG induced the vagus afferent activation in most of the brain during the infusion period. In contrast, intragastric infusion of $60 \mathrm{mM}$ glucose induces long-lasting activation for more than $1 \mathrm{~h}$. These different temporal and regional activation patterns in the brain are due to distinctive signaling pathways between the gut and brain and they result in distinctive effects on postingestive behavior.

\section{Signaling mechanisms of the gut-brain axis promotes control of metabolism for homeostasis}

Ingested food is digested and absorbed in free form as small molecular nutrients in the GI tract. The afferent
Fig. 4 Mean intake of conditioned stimulus (filled bar $\mathrm{CS}+$, flavored water with intragastric infusion of MSG, $\mathrm{NaCl}$, or glucose; and open bar $\mathrm{CS}-$, flavored water with intragastric infusion of water) solutions in the pre-test and test periods. Mean percent intakes of the $\mathrm{CS}+$ solution are shown above the bars. Reproduced from Uematsu et al. [3]

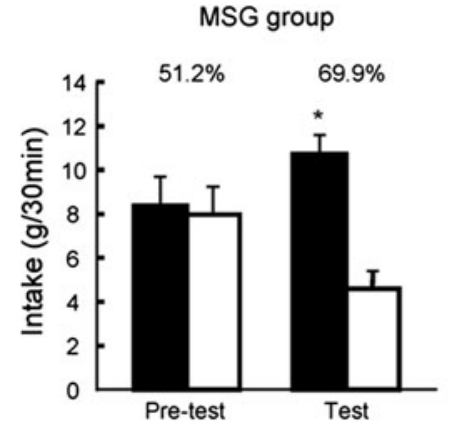

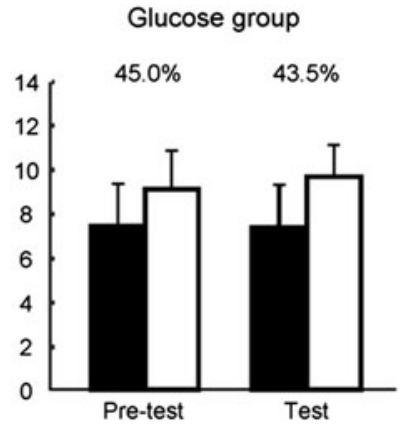


Fig. 5 Chronological variation of the percent changes in significantly activated area in rats. Horizontal axis is elapsed time after the onset of infusion. Reproduced from Tsurugizawa et al. [37]

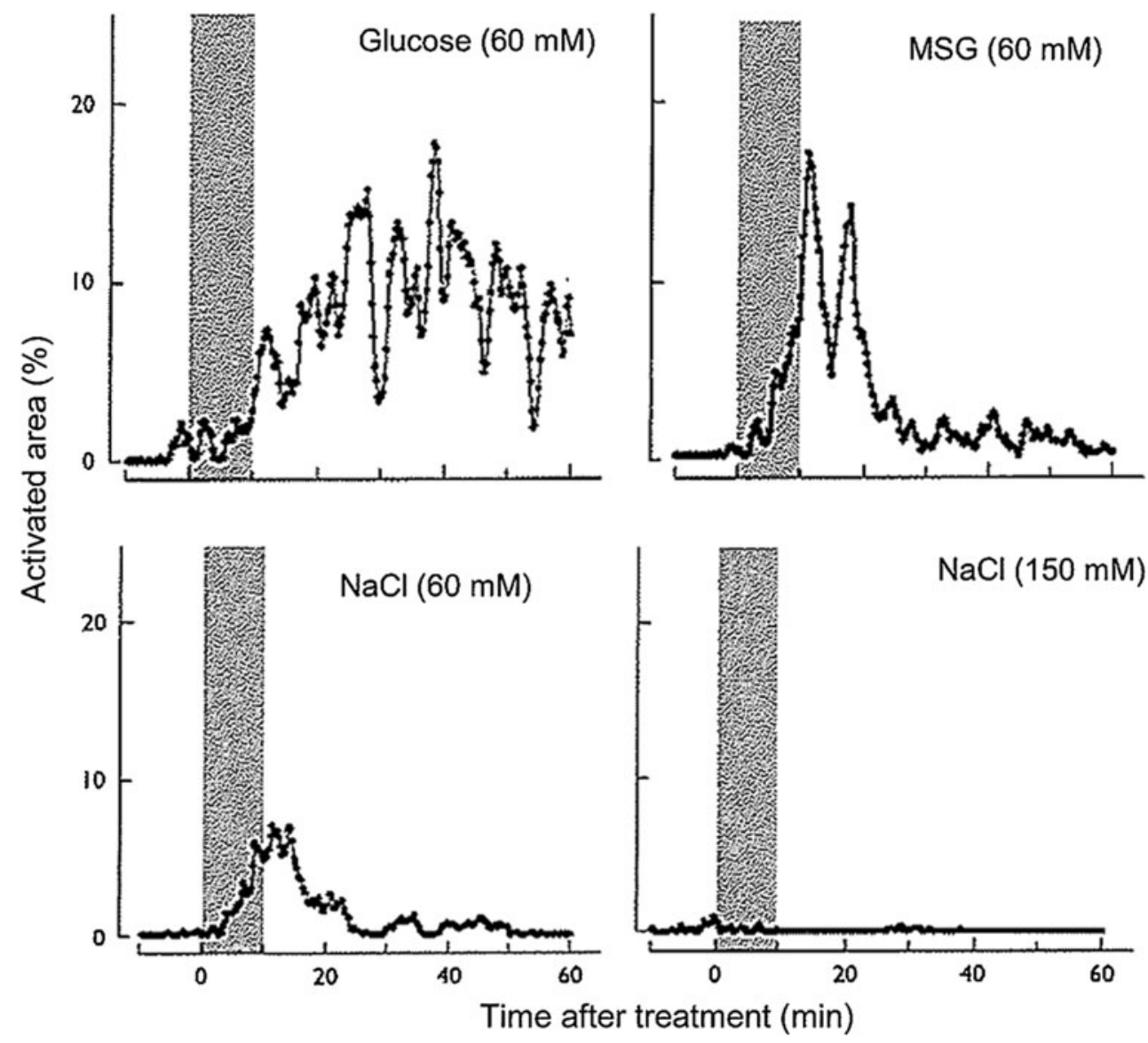

vagus nerve, which innervates the entire GI tract and projects to the nucleus of the solitary tract (NTS), is activated by each of these free form nutrients during digestion processes. In parallel, peripheral humoral factors such as insulin and GLP-1 are released. In addition to the process of absorption and metabolism in the gut, recent studies have indicated that the stomach, duodenum, and other small intestine express to localize chemosensing taste receptors in the luminal layer, and other G-protein-coupled receptors (GPCRs). The T1R receptors, which are responsible for the chemoreception of the sweet and umami tastes, and the family of T2R receptors, which mediate the chemoreception of the bitter taste, are both expressed in the gut $[46,47]$. A fatty acid receptor, GPR120, exists in both the oral cavity and the GI tract, and these acids interact with it to induce the release of circulating GLP-1 [48]. Furthermore, free fatty acids also interact with another receptor, GPR40, in the GI tract to promote secretion of GLP-1 [49] and cholecystokinin (CCK) [50]. The GLP-1 and CCK evoke c-fos positive immunoreactivity in several brain regions, including the amygdala [51-53]. Intragastric infusion of glucose solution increases blood glucose levels, GLP-1, and insulin. Circulating GLP-1 also acts on neurons in the NTS. Recently, we demonstrated that fluctuations in insulin following the intragastric administration of glucose correlate with fMRI responses in the amygdala, the ventromedial hypothalamus, and the nucleus accumbens [38].

Electrophysiological studies have shown that intragastric and enteric delivery of amino acids and lipids both activate the afferent vagus nerve as described above [30$32,54,55]$. Intraportal administration of amino acids also activates vagal afferent responses [56]. These reports indicate that the vagus afferent response is important for the transmission of each dietary nutrient information in the gut to the brain. Interestingly, behavioral studies have shown that abdominal vagotomy eliminates CFP in response to intragastric infusion of MSG [4] but does not affect CFP response in the case of carbohydrates in rats [57]. An fMRI study showed that total and partial abdominal vagotomy diminished Glu-induced activation in the NTS and the hypothalamus, whereas total vagotomy did not affect glucose-induced brain activation [38]. Instead, brain activation was correlated with fluctuations in insulin following intragastric glucose infusion [38]. These results from fMRI studies in vagotomized rats are consistent with postingestive behavior studies, indicating that internal signals in response to Glu mainly involve the vagus nerve, whereas those in response to glucose at least partially involve insulin release. Finally, there are distinct postingestive effects in response to different nutrients, 
resulting in the activation of forebrain regions. The spatial and temporal patterns of brain activation could link with postingestive behavioral and physiological effects underlying the maintenance of homeostasis following a meal.

\section{Glutamate plays a role to promote digestive juice secretion and bowel movement}

Weaning is a critical period for infants, because of changes of breast milk to foods exposing the infant to a risky environment including pathogenic infections. Recently, we analyzed free glutamate content in the pancreatic juice several fold higher than the plasma level. The localization of free glutamate in the pancreas using a specific antibody was seen so that zymogen granule in the acinar cells was positive. During exocytosis of the zymogen granule as digestive juices, also free glutamate is to be released. Other acinar cell membranes expresses mGluR1 to receive free glutamate stimuli due to exocytosis of zymogen granules. The cascade type of pancreatic juice secretion should be triggered by released free Glu in the pancreas. Similar localization of free glutamate is in the pancreatic islet. These endocrine cells also express metabotropic glutamate receptors type 4 and 8 receiving free Glu stimuli released from the vagus nerve-end like cephalic phase stimuli [59]. So, exocrine and endocrine systems to release digestive enzymes as well as pancreatic hormones have a common fashion in the digestive organs to be controlled by free glutamate stimuli. This free glutamate is originated from glutamine (Glu) in body fluid. Intracellular glutamine is enzymatically metabolized to glutamate and yielded free glutamate to be incorporated into the zymogen granules. The free form of glutamate in the digestive juice directly stimulate the alimentary tract to regulate digestion and absorption normally, through $\mathrm{pH}$ control and mucin release for the barrier system. The capacity of biosynthesis of free glutamate is immature in infants and retarded in the elderly. So, the propriate level of dietary glutamine as a precursor of glutamate and free glutamate itself are beneficial for normal digestive function. We tried to use piglets similar to a human infants model so that just weaned piglets are anorexigenic is concomitant with negative energy balance. Furthermore, the piglets' immune systems seem to be insufficient after weaning. They used to display diarrhea and subsequent dehydration, and subsequently malnutritional piglet growth is to be retarded. From the point of digestive physiology, switching from a milk formula to a solid diet negatively impacts the epithelium of the intestine. As described above, Glu has a potential for the intestine to release mucin due to establishment of a barriers system against microbiota. Therefore, supplementation liquid for weaning piglets containing Glu and L-glutamine
(Gln) as a precursor for Glu adding to the weaning diet results in a significant improvement of the diarrhea index and their state of nutrition. Similar cases are often observed in the elderly. Habituation studies employed elderly subjects to be offered rice glue containing 0.5 (w/v) MSG for 3 months. Double blind and cross-over studies yield improvement in the quality of life concerning emotion and appetite for foods, reduced incidence of diarrhea and vomiting, peripheral circulation to cure bed thaw lesion, etc. reflecting the basic metabolic rate enhanced by Glu signaling in gut [59].

\section{Conclusions}

Free Glu signaling during a meal and subsequent processes in the alimentary organs plays important physiological roles in the perception of umami taste, visceral information and regulation of endo-and exocrine systems, and excitatory neurotransmission. But there are no way to entry dietary free Glu into the circulating blood as well as brain cerebrospinal fluid because of both the intestinal hepatic barrier and the blood brain barrier are not permeable for free Glu. Glu signaling by dietary Glu is positively effective in the oral cavity through alimentary tracts via several receptors. In our series of studies reviewed here, we showed that dietary Glu also stimulates Glu sensors in the stomach and intestines, producing local effects on gut function for ingested food digestion. Moreover, via the release of signaling molecules NO and 5-HT, the presence of Glu in the gut leads to the activation of the vagal afferent nerve which in turn modulates a number of target areas in the brain, particularly the gastric vagal afferent responds to Glu alone rather than electrolytes, sugars and other amino acids, suggesting that Glu signaling in the stomach is to be a signal for food intake controlling whole processes of digestion and absorption of yielded nutrients. In addition, we described the postingestive effects of Glu and showed that they were different from those elicited by glucose and lipids. Previous fMRI observation in the brain and behavioral studies in rodents have indicated that Glu has positive postingestive effects through the vagal afferent nerve that controls appetite and satiety for food. However, Glu does not have the same reinforcing properties of craving and/or addictive substances such as sugars and alcohol. Altogether, these findings indicate that dietary Glu influences numerous physiological functions, suggesting a broad, integrative role for dietary Glu in body homeostasis. This newly developed method for brain functional change observation in well-trained awake rats using a fMRI after nutrient stimulation in the gut by gastric intubation into the stomach was recently confirmed by c-fos expression in similar rats, suggesting that gut-brain communication 
through chemical senses of nutrient, particularly Glu, becomes available to research using awake animals in connection with non-invasive treatment $[39,58]$ due to an understanding why rats, fed a high-fat and high-sugar diet, do not become obese when Glu solution is available to drink with this food intake through higher thermogenesis due to energy expenditure. Dietary Glu and glutamine as a precursor for Glu signaling are positively promoted as a function of the alimentary tracts in infants as well as the elderly because of the biosynthesis capacity of free Glu is not sufficient in the exo- and endocrine organs [60].

Acknowledgments We would like to take this opportunity to thank Drs. Makoto Bannai, Akihiko Kitamura and Tomokazu Tsurugizawa for their substantial contribution to our research outcome. We also appreciate Dr. Gary K. Beauchamp who provided editorial advice.

Conflict of interest The authors declare that they have no conflict of interest.

\section{References}

1. Giacometti T. Free and bound glutamate in natural products. In: Filer Jr LJ, Garattini MR, Kare MR, Reynolds WA, Wurtman RJ, editors. Glutamic acid: advances in biochemistry and physiology. New York: Raven Press; 1979. p. 25-34.

2. Young VR, Ajami AM. Glutamate: an amino acid of particular distribution. J Nutr. 2000;130(Suppl 4S):892S-900S.

3. Uematsu A, Tsurugizawa T, Kondoh T, Torii K. Conditioned flavor preference learning by intragastric administration of L-glutamate in rats. Neurosci Lett. 2009;451:190-3.

4. Uematsu A, Tsurugizawa T, Uneyama H, Torii K. Brain-gut communication via vagus nerve modulates conditioned flavor preference. Eur J Neurosci. 2010;31:1136-43.

5. Hector MP. Reflexes of salivary secretion. In: Garrett JR, Ekström J, Anderson LC, editors. Neural mechanisms of salivary gland secretion. Basel: Karger; 1999. p. 196-217.

6. Hodson NA, Linden RWA. The effect of monosodium glutamate on parotid salivary flow in comparison to the response to representatives of the other four basic tastes. Physiol Behav. 2006;89:711-7.

7. Niijima A. Control of liver function and neuroendocrine regulation of blood glucose levels. In: Brooks CMcC, Koizumi K, Sato A, editors. Integrative functions of the autonomic nervous system. Tokyo/Elsevier: University of Tokyo Press and Amsterdam/ North-Holland Biomedical Press; 1979. p. 68-83.

8. Niijima A. Effects of taste stimulation on the efferent activity of the pancreatic vagus nerve in the rat. Brain Res Bull. 1991;26: 161-4.

9. Niijima A. Effects of taste stimulation on the efferent activity of the autonomic nerves in the rat. Brain Res Bull. 1991;26:165-7.

10. Niijima A. Effect of umami taste stimulations on vagal efferent activity in the rat. Brain Res Bull. 1991;27:393-6.

11. Louis-Sylvestre J. Preabsorptive insulin release and hypoglycemia in rats. Am J Physiol. 1976;230:56-60.

12. Steffens AB. Influence of the oral cavity on insulin release in the rat. Am J Physiol. 1976;230:1411-5.

13. Strubbe JH, Steffens AB. Rapid insulin release after ingestion of a meal in the unanesthetized rat. Am J Physiol. 1975;229: 1019-22.
14. Jiang ZY, Niijima A. Effects of taste stimuli on the efferent activity of the gastric vagus nerve in rats. Neurosci Lett. 1986;69:42-6.

15. Ikuno H, Sakaguchi T. Gastric vagal functional distribution in the secretion of gastric acid produced by sweet taste. Brain Res Bull. 1990;25:429-31.

16. Niijima A. Effects of oral and intestinal stimulation with umami substance on gastric vagus activity. Physiol Behav. 1991;49: $1025-8$.

17. Niijima A. Reflex effects of oral, gastrointestinal and hepatoportal glutamate sensors on vagal nerve activity. J Nutr. 2000; 130:971S-3S.

18. Niijima A, Togiyama T, Adachi A. Cephalic-phase insulin release induced by taste stimulus of monosodium glutamate (umami taste). Physiol Behav. 1990;48:905-8.

19. Tonosaki K, Hori Y, Shimizu Y, Tonosaki K. Relationships between insulin release and taste. Biomed Res. 2007;28:79-83.

20. deFonseka A, Kaunitz J. Gut sensing mechanisms. Curr Gastroenterol Rep. 2009;11:442-7.

21. Kitamura A, Niijima A, Torii K, Uneyama H. Amino acidsensing by the abdominal vagus. In: Costa A, Villalba E (eds) Horizons in neuroscience research, vol. 1. New York: Nova Science Publishers, Inc.; 2010. p. 367-77.

22. Raybould HE, Glatzle J, Freeman SL, Whited K, Dracel N, Liou A, Bohan D. Detection of macronutrients in the intestinal wall. Auton Neurosci. 2006;125:28-33.

23. Rozengurt E, Sternini C. Taste receptor signaling in the mammalian gut. Curr Opin Pharmacol. 2007;7:557-62.

24. Uneyama H, Niijima A, San Gabriel A, Torii K. Luminal amino acid sensing in the rat gastric mucosa. Am J Physiol Gastrointest Liver Physiol. 2006;291:G1163-70.

25. Fujita T, Kobayashi S, Yui R. Paraneuron concept and its current implications. Adv Biochem Psychopharmacol. 1980;25:321-5.

26. Höfer D, Püschel B, Drenckhahn D. Taste receptor-like cells in the rat gut identified by expression of alpha-gustducin. Proc Natl Acad Sci USA. 1996;93:6631-4.

27. Kitamura A, Sato W, Uneyama H, Torii K, Niijima A. Effects of the intragastric infusion of inosine monophosphate and L-glutamate on the vagal gastric afferent activity and subsequent autonomic reflexes. J Physiol Sci. 2011;61(1):65-71.

28. Sharma KN, Nasset ES. Electrical activity in mesenteric nerves after perfusion of gut lumen. Am J Physiol. 1962;202:725-30.

29. Jeanningros R, Mei N. Données préliminaires sur la réponse des chémorécepteurs intestinaux acides aminés. Reprod Nutr Dév. 1980;20:1615-9.

30. Jeanningros R. Vagal unitary responses to intestinal amino acid infusions in the anesthetized cat: a putative signal for protein induced satiety. Physiol Behav. 1982;28:9-21.

31. Niijima A, Torii K, Uneyama $H$. Role played by vagal chemical sensors in the hepato-portal region and duodeno-intestinal canal: an electrophysiological study. Chem Senses. 2005;30(Suppl 1): i178-9.

32. Eastwood C, Maubach K, Kirkup AJ, Grundy D. The role of endogenous cholecystokinin in the sensory transduction of luminal nutrient signals in the rat jejunum. Neurosci Lett. 1998;254:145-8.

33. Schwartz GJ, Moran TH. Duodenal nutrient exposure elicits nutrient-specific gut motility and vagal afferent signals in rat. Am J Physiol Regul Integr Comp Physiol. 1998;274:R1236-42.

34. Raybould HE. Sensing of glucose in the gastrointestinal tract. Auton Neurosci. 2007;133:86-90.

35. Mei N. Intestinal chemosensitivity. Physiol Rev. 1985;65: 211-37.

36. Schwartz GJ. The role of gastrointestinal vagal afferents in the control of food intake: current prospects. Nutrition. 2000;16: 866-73. 
37. Tsurugizawa T, Kondoh T, Torii K. Forebrain activation induced by postoral nutritive substances in rats. NeuroReport. 2008;19:1111-5.

38. Tsurugizawa T, Uematsu A, Nakamura E, Hasumura M, Hirota M, Kondoh T, Uneyama H, Torii K. Mechanisms of neural response to gastrointestinal nutritive stimuli: the gut-brain axis. Gastroenterology. 2009;137:262-73.

39. Tsurugizawa T, Uematsu A, Uneyama H, Torii K. Blood oxygenation level-dependent response to intragastric load of corn oil emulsion in conscious rats. NeuroReport. 2009;20:1625-9.

40. Kondoh T, Torii K. MSG intake suppresses weight gain, fat deposition and plasma leptin levels in male Sprague-Dawley rats. Physiol Behav. 2008;95:135-44.

41. Sclafani A. Fat and sugar flavor preference and acceptance in C57BL/6J and 129 mice: experience attenuates strain differences. Physiol Behav. 2007;90:602-11.

42. Ackroff K, Sclafani A. Flavor preferences conditioned by intragastric infusion of ethanol in rats. Pharmacol Biochem Behav. 2001;68:327-38.

43. Touzani K, Sclafani A. Lateral hypothalamic lesions impair flavour-nutrient and flavour-toxin trace learning in rats. Eur $\mathbf{J}$ Neurosci. 2002;16:2425-33.

44. Mark GP, Smith SE, Rada PV, Hoebel BG. An appetitively conditioned taste elicits a preferential increase in mesolimbic dopamine release. Pharmacol Biochem Behav. 1994;48:651-60.

45. Shibata R, Kameishi M, Kondoh T, Torii K. Bilateral dopaminergic lesions in the ventral tegmental area of rats influence sucrose intake, but not umami and amino acid intake. Physiol Behav. 2009;96:667-74.

46. Rozengurt E. Taste receptors in the gastrointestinal tract. I. Bitter taste receptors and alpha-gustducin in the mammalian gut. Am J Physiol Gastrointest Liver Physiol. 2006;291:G171-7.

47. Dyer J, Salmon KS, Zibrik L, Shirazi-Beechey SP. Expression of sweet taste receptors of the T1R family in the intestinal tract and enteroendocrine cells. Biochem Soc Trans. 2005;33:302-5.

48. Hirasawa A, Tsumaya K, Awaji T, Katsuma S, Adachi T, Yamada $\mathrm{M}$, et al. Free fatty acids regulate gut incretin glucagon-like peptide-1 secretion through GPR120. Nat Med. 2005;11:90-4.

49. Itoh Y, Kawamata Y, Harada M, Kobayashi M, Fuji R, Fukusumi $\mathrm{S}$, et al. Free fatty acids regulate insulin secretion from pancreatic beta cells through GPR40. Nature. 2003;422:173-6.
50. Beardshall K, Frost G, Morarji Y, Domin J, Bloom SR, Calam J. Saturation of fat and cholecystokinin release: implications for pancreatic carcinogenesis. Lancet. 1989;2:1008-10.

51. Viltart O, Sartor DM, Verberne AJ. Chemical stimulation of visceral afferents activates medullary neurones projecting to the central amygdale and periaqueductal grey. Brain Res Bull. 2006;71:51-9.

52. Wang L, Martinez V, Barrachina MD, Tache Y. Fos expression in the brain induced by peripheral injection of CCK or leptin plus CCK in fasted lean mice. Brain Res. 1998;791:157-66.

53. Baggio LL, Huang Q, Brown TJ, Drucker DJ. A recombinant human glucagon-like peptide (GLP)-1-albumin protein (albugon) mimics peptidergic activation of GLP-1 receptor-dependent pathways coupled with satiety, gastrointestinal motility, and glucose homeostasis. Diabetes. 2004;53:2492-500.

54. Mei N, Garnier L. Osmosensitive vagal receptors in the small intestine of the cat. J Auton Nerv Syst. 1986;16:159-70.

55. Randich A, Tyler WJ, Cox JE, Meller ST, Kelm GR, Bharaj SS. Responses of celiac and cervical vagal afferents to infusions of lipids in the jejunum or ileum of the rat. Am J Physiol Regul Integr Comp Physiol. 2000;278:R34-43.

56. Niijima A, Meguid MM. An electrophysiological study on amino acid sensors in the hepato-portal system in the rat. Obes Res. 1995;3(Suppl 5):741S-5S.

57. Sclafani A, Lucas F. Abdominal vagotomy does not block carbohydrate-conditioned flavor preferences in rats. Physiol Behav. 1996;60:447-53.

58. Otsubo H, Kondoh T, Shibata M, Torii K, Ueta Y. Induction of Fos expression in the rat forebrain after intragastric administration of monosodium L-glutamate, glucose and $\mathrm{NaCl}$. Neuroscience. 2011;196:97-103.

59. Murayama A, Uehara A, Yatsushiro S, Echigo N, Morimoto R, Morita M, et al. A novel variant of ionotropic glutamate receptor regulates somatostatin secretion from g-cell of islets of Langerhans. Diabetes. 2004;53:1743-53.

60. Yamamoto S, Tomoe M, Toyama K, Kawai M, Uneyama H. Can dietary supplementation of monosodium glutamate improve the health of the elderly? Am J Clin Nutr. 2009;90(3):97-103. 